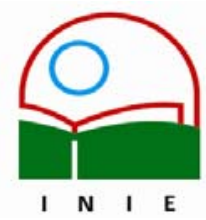

Actualidades Investigativas en Educación

Revista Electrónica publicada por el

Instituto de Investigación en Educación

Universidad de Costa Rica

ISSN 1409-4703

http://revista.inie.ucr.ac.cr

COSTA RICA

\title{
TEORÍAS DE NIÑAS Y NIÑOS SOBRE EL CASTIGO PARENTAL. APORTES PARA LA EDUCACIÓN Y LA CRIANZA
}

THEORIES OF GIRLS AND BOYS ABOUT PARENTAL PUNISHMENT. CONTRIBUTIONS FOR EDUCATION AND CHILD-RAISING PRACTICES

\author{
Volumen 9, Número 2 \\ pp. 1-29
}

Este número se publicó el 30 de agosto 2009

Ginette Sánchez Gutiérrez

La revista está indexada en los directorios:

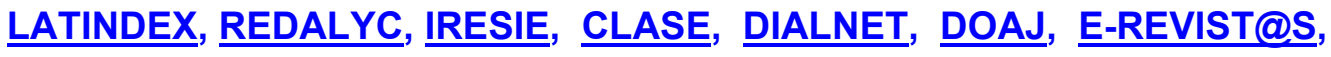

La revista está incluida en los sitios:

REDIE, RINACE, OEI, MAESTROTECA, PREAL, HUASCARAN, CLASCO 


\title{
TEORÍAS DE NIÑAS Y NIÑOS SOBRE EL CASTIGO PARENTAL. APORTES PARA LA EDUCACIÓN Y LA CRIANZA THEORIES OF GIRLS AND BOYS ABOUT PARENTAL PUNISHMENT. CONTRIBUTIONS FOR EDUCATION AND CHILD-RAISING PRACTICES
}

\section{Ginette Sánchez Gutiérrez ${ }^{1}$}

\begin{abstract}
Resumen: El presente estudio cualitativo, correspondiente a una tesis de grado en psicología, expone las teorías de niñas y niños escolares sobre las prácticas de castigo parental a partir del modelo teórico del desarrollo moralemocional. Mediante los aportes del Método de Comparación Constante (o Teoría Fundamentada) se elaboró un instrumento adecuado a la población del estudio, al tema y según algunos criterios ético-metodológicos para finalmente, realizar entrevistas individuales a partir de estímulos visuales y narrativos.

Como conclusión general, se encontró una pluralidad de sentidos y perspectivas sobre las prácticas de castigo parental, posible como teoría, desde el sentido común, debido a la capacidad infantil de dimensionar la posición adulta y el contexto global de las situaciones desde una actitud empática que derivó en razonamientos y explicaciones con contenido cognitivo-afectivo.
\end{abstract}

Palabras clave: PSICOLOGÍA DEL DESARROLLO, DESARROLLO MORAL-EMOCIONAL INFANTIL, CASTIGO PARENTAL, CRIANZA EN COSTA RICA, TEORIIAS INFANTILES, EMOCIONES MORALES.

\begin{abstract}
This qualitative study, corresponding to a graduate thesis in Psychology, details the theories of primary school girls and boys about parental punishment from a moral-emotional theoretical model of development. Through the use of the Constant Comparison Method (or Grounded Theory), an instrument was designed that could fit the subjects, the topic and some ethical and methological criteria, in order to finally carry out individual interviews based on visual and narrative stimuli.

As a general conclusion, a plurality of meanings and perspectives about parental punishment was found: a possible commonsense theory about child's capacity to visualize the adult position and the global context resulted in empathetic ideas and explanations of a cognitive-affective nature.
\end{abstract}

Key Words: DEVELOPMENTAL PSYCHOLOGY, MORAL-EMOTIONAL DEVELOPMENT, PARENTAL PUNISHMENT, CHILD RAISING IN COSTA RICA, INFANTILE THEORIES, MORAL EMOTIONS.

\section{Introducción}

La problemática de la niñez tiene, en la actualidad, gran relevancia en las políticas públicas, principalmente a raíz de la ratificación internacional que hicieran más de 190 Estados Partes, incluido el nuestro, sobre la Convención sobre los Derechos del Niño (Fundación Save The Children, s.f.) Este reciente interés, de atención y protección, hacia la población infantil y adolescente no es casual, obedece a una larga data de historias de maltrato y abusos en su contra.

\footnotetext{
${ }^{1}$ Licenciada en Psicología, Universidad de Costa Rica. Labora actualmente como docente en la Escuela de Psicología y en la Revista Káñina de la Universidad de Costa Rica.
}

Dirección electrónica: ginettecr@yahoo.com

Artículo recibido: 16 de enero, 2009

Aprobado: 24 de julio, 2009 
Revisando un pasado histórico que nos compete como cultura, en sociedades precristianas como Grecia y Roma antiguas, se ha encontrado documentada la agresión contra las niñas y los niños; no obstante, en esta época no se concebía la existencia de malos tratos ni se detectaba preocupación por estos (Permuy y Buceta, 1995). Esto explica, en parte, cómo el recurso a la violencia como práctica disciplinaria ha sido utilizado ampliamente, gracias a su legitimación social en una cultura patriarcal que visualiza a la infancia como un objeto propiedad del adulto (tal vez una distorsión del recurso de la patria potestad), lo cual ha justificado ampliamente el uso de la violencia como pedagogía de crianza o pedagogía negra siguiendo a Allice Miller (1998), en tanto el niño o la niña no ha tenido un papel protagónico en las decisiones sobre sus propias necesidades, intereses y autonomía.

La Revolución Industrial (siglo XIX) despierta la conciencia por legislar y proteger a la infancia, a raíz de la explotación laboral que sufrían las niñas y los niños en diferentes trabajos forzados, aprovechando su juventud como fuerza de trabajo valiosa pues, con sus pequeños cuerpos y una menor necesidad de alimento,se les podían exigir tareas especialmente difíciles (Vargas, 1992). De ahí una concepción de niñez como de menor categoría que la de adultez, visible en expresiones como "los menores" versus los "mayores", los "pequeños" versus "los grandes".

Posteriormente (siglo XX), gracias a los estudios pioneros de Tardieu, Athol Jonson y Henry Kempe, se acuña el término científico "Síndrome del niño golpeado" y se inician los análisis relativos a las consecuencias del maltrato infantil (Permuy y Buceta, 1995). Esto abrió el portal a la consideración de los niños y las niñas como sujetos de derecho, lo cual derivó finalmente en la promulgación de la convención, en un intento por dar una respuesta legal que tuviera incidencia en lo social para hacer frente al problema del maltrato infantil.

\section{Concepción de niñez en Costa Rica}

En nuestro país, una concepción de niñez, llamada adultocéntrica, ha permeado, hasta la fecha, el trato a la población infantil y adolescente. Esto se expresa en las prácticas sociales de múltiples instancias: jurídicas, institucionales, sociales y familiares. Por otro lado, en las últimas décadas se ha generado un proceso reflexivo en estas mismas instancias. Algunos ejemplos: en el ámbito jurídico, el Código de la Niñez y la Adolescencia alerta sobre el interés superior del niño, la "obligación de procurar el desarrollo integral de la persona 
menor de edad" que "corresponde, en forma primordial, a los padres o encargados" (República de Costa Rica, Código de la Niñez y la Adolescencia, Ley № 7739, artículos 5 y 7) y el deber del Estado de adoptar las medidas administrativas, legislativas, presupuestarias y de cualquier índole, que garanticen la plena efectividad de sus derechos; en el ámbito institucional, el Patronato Nacional de la Infancia, como entidad rectora en niñez y adolescencia, promueve un paso cualitativo, aún incipiente pero progresivo, desde lo que se ha llamado la Doctrina de la Situación Irregular, en la que el niño o la niña es un "menor" objeto de políticas de corte asistencialista (con énfasis en cubrir necesidades básicas), a una Doctrina de Protección Integral, que modifica la óptica hacia la concepción de sujeto, ciudadano activo, con autonomía progresiva, en razón del desarrollo natural, con derechos, deberes y necesidades a las que hay que responder, pero sin dejar de lado a la persona y su condición de ciudadano.

La Doctrina de la Protección Integral pretende evolucionar la concepción de niñez de una visión de objeto a una de sujeto, donde se considere a las niñas y los niños como ciudadanos pensantes y merecedores de un trato digno y respetuoso. No obstante, las prácticas sociales y familiares actuales no reflejan a cabalidad este cambio, pues en el caso de Costa Rica todavía son constantes las agresiones hacia estas personas, habiéndose encontrado como prácticas disciplinarias más frecuentes el uso del castigo físico y el maltrato verbal (Zúñiga y Carballo, 2003).

Por otra parte, el VI Informe del Estado de los Derechos de la Niñez y la Adolescencia en Costa Rica (Universidad de Costa Rica - UNICEF, 2008), apunta que si bien ha habido un avance en la participación plena de esta población con respecto a sus derechos, aún los cambios que se han gestado a nivel macrosocial (jurídico e institucional principalmente) en el Sistema Nacional de Protección Integral de los Derechos de la Niñez y la Adolescencia, tienen problemas estructurales y se han enfocado en problemáticas más agudas, a saber, maltrato, abuso y explotación sexual infantil, lo cual ha dejado de lado la promoción de integralidad, que debe reflejarse en las prácticas sociales de la cotidianidad (familia, escuela, vecindario, comunidad), claro está, sin descuidar las mencionadas problemáticas vigentes.

Actualmente, señala el VI EDNA,

la arquitectura del SNPI no se ha completado por existir vacíos y ausencias en los planos normativo, organizacional y financiero. Los avances en el funcionamiento, logros e impacto del SNPI son aún de una escala pequeña y se reconoce, por ello, 
como un desafío para el país, el desarrollo de las dimensiones y expresiones regionales y locales de este. (UNICEF, 2008, p. 8)

De cara a esta realidad, el estudio sobre las teorías infantiles del castigo parental responde al interés por evidenciar lo que piensan y sienten niñas y niños costarricenses sobre la realidad de crianza presentada por las figuras adultas más cercanas, cuando en muchos casos se recurre a formas violentas de trato, con una supuesta pretensión educativa, lo cual va en detrimento del disfrute pleno de sus derechos humanos.

\subsection{El punto de partida}

Al explorar las teorías infantiles sobre las prácticas de castigo parental, en este estudio de grado en psicología, se planteó como premisa básica que las niñas y los niños elaboran explicaciones e interpretaciones propias (de contenido cognitivo, afectivo y moral) sobre la realidad que les muestran las figuras parentales, cuya función en la socialización primaria de comportamientos, valores y actitudes esperados y promovidos es una tarea que se manifiesta permanentemente en la cotidianidad de la crianza.

Esta investigación enfatizó en el razonamiento o comprensión moral emocional ${ }^{2}$ de seis niñas y seis niños escolares, con edades entre los diez y los doce años, sobre las prácticas de castigo que utilizan las figuras parentales o cuidadores en el entorno familiar o íntimo. El objetivo general fue elaborar una propuesta de comprensión de una teoría infantil desde el sentido común, sobre las prácticas de castigo parental. Al momento de la investigación, los participantes cursaban el segundo ciclo de la Educación General Básica en una escuela pública urbana ubicada del cantón de Desamparados. El estudio se realizó durante los años 2006 y 2007.

De acuerdo con la revisión de antecedentes, se encontró una ausencia en nuestro país de investigación relativa a la comprensión infantil moral-emocional sobre las prácticas de castigo parental; de esta forma, la investigación contribuye a ampliar el conocimiento científico sobre la dinámica intersubjetiva presente en la utilización de las diferentes prácticas

\footnotetext{
${ }^{2}$ El razonamiento moral emocional ha sido estudiado por diversos autores a partir de la comprensión infantil de dilemas morales y situaciones cotidianas. Implica la realización de inferencias y abstracciones de tipo cognitivo y afectivo sobre los protagonistas de las situaciones y el contexto de los implicados, lo cual deriva en atribuciones con contenido moral emocional sobre las intenciones y actuaciones de las personas que interactúan en esos eventos. (Sánchez, 2007, pp. 15-16, 33, 36, 37,39)
} 
de castigo, queriendo indagar cómo son entendidas y asimiladas estas medidas correctivas en el grupo de participantes.

Como aporte a la práctica profesional de la Psicología, se incluye la perspectiva infantil para brindar alternativas y soluciones hacia una lectura crítica de las prácticas de castigo, conducente a proponer mecanismos efectivos a las necesidades del desarrollo de este importante sector de nuestra sociedad, todo ello sustentados desde un enfoque de derechos y respetuosos del entendimiento de la realidad, tal y como la conciben las niñas y los niños.

\section{Teorías infantiles sobre el castigo parental}

La capacidad de pensamiento y atribución emocional de las niñas y los niños ha sido estudiada en diversos escenarios de la vida cotidiana, incluido el familiar, el escolar y el comunitario (Piaget, 1932/1974; Kohlberg, 1967/1969; Wellman, 1995; Nunner-Winkler y Sodian, 1988, etc). En relación con esto, la referencia sobre teorías infantiles del sentido común se sustenta en la idea de una capacidad progresiva del pensamiento concreto al abstracto que se desarrolla desde edades muy tempranas, como respuesta a los retos de la vida cotidiana. Esta inteligencia activa posibilita la comprensión infantil de los estados mentales y volitivos; es decir, la discriminación de intenciones, valores, creencias, deseos y afectos (Wellman, 1995). Asimismo, existe una atribución moral y emocional (NunnerWinkler y Sodian, 1988) que entra en juego en la relación vincular cuando se introduce el castigo, y los hijos e hijas participan en el sentido que le otorgan a la situación que fue merecedora de alguna medida punitiva o correctiva. Esta atribución variará según las particularidades de la situación, de los protagonistas, de las consecuencias de las acciones consideradas incorrectas, del tipo de castigo estipulado y de la intención y función que sea percibida.

El castigo parental ha sido definido, desde un punto de vista unilateral y centrado en la persona adulta, como un factor que media la interacción entre padres, madres y sus hijas e hijos en situaciones particulares de conflicto. En términos generales consiste en una modalidad del repertorio de estrategias educativas, de corrección o amonestación que utilizan madres y padres para formar a sus hijas e hijos; pretende socializar al infante, mediante restricciones y amonestaciones (Valtin y Walter, 1996), en los valores, creencias y comportamientos esperados, en particular, la obediencia, el respeto y el buen 
comportamiento (Keller, Kuensemueller, Abels, Voelker, Yovsi, Jensen, Papaligoura, Lohaus, Rosabal-Coto, Kulks y Mohite, 2005).

Las prácticas de castigo se inscriben en la cotidianidad del vínculo primario con las figuras parentales, mediatizan el vínculo de apego y operan como un mecanismo regulador de las emociones morales ${ }^{3} y$ el comportamiento (Eisenberg, Spinrad, Losoya y Fabes, 1999). Desde este vínculo de apego, el castigo parental se presenta cuando existe una diferencia en la valoración adulta y la infantil respecto a una acción realizada por el niño o la niña, y la madre o el padre asume que su perspectiva es la correcta; por lo tanto, se debe corregir al hijo o a la hija por medio del castigo.

No obstante, desde la perspectiva infantil, aun cuando el castigo es adjudicado por la figura adulta, se escenifica en un contexto global que es asimilado por el niño o la niña desde su propia lógica, la cual incluye aspectos de índole cognitivo, moral y emocional interrelacionados. En este proceso de asimilación, la comprensión de lo ocurrido por parte de las niñas y los niños tendrá que pasar por un proceso complejo de toma de rol y empatía (Eisenberg, Shea, Carlo y Knight, 1991; Thoits, 1989; Reyes, 2006), es decir, por un desdibujamiento del ser hijo o hija, para dimensionar la figura adulta y ponerse en su lugar, y desde allí, tratar de entender por qué y para qué fue necesario el castigo. Esta toma de rol implica que, incluso en ausencia de la persona física, el niño o la niña abstrae qué pudo haber pensado y sentido el otro ante la situación y sus consecuencias.

En otro sentido, el recurso a la empatía se dificulta cuando los castigos aplicados responden más a una reacción emocional irreflexiva y sin explicación lógica para el niño o la niña. En este segundo caso, se habla de una asimilación forzada, con posibles consecuencias emocionales negativas, en virtud de no contar con un ambiente propicio para la reflexión y aprendizaje de lo sucedido.

\footnotetext{
3 También llamadas emociones autoconscientes (self conscious emotions), consisten en reacciones emocionales inducidas de forma vicaria por interacción con reacciones cognitivas, de forma tal que la relación entre procesos cognitivos y emociones morales no es unidireccional y tiende a ser recíproca e interrelacionada, entendiéndose entonces que los procesos cognitivos condicionan e inducen emociones morales y viceversa. Entre las emociones morales se identifican la empatía, la simpatía y el malestar personal, así como las emociones orientadas hacia sí mismo en contraposición a las emociones altruistas. (Sánchez, 2007: 41-42) Volumen 9, Número 2, Año 2009, ISSN 1409-4703
} 


\section{Metodología}

\subsection{Tipo de estudio}

Esta investigación consistió en un estudio cualitativo descriptivo-comprensivo, basado en algunos de los principios de la Metodología de Comparación Constante (MCC) o Teoría Fundamentada (Grounded Theory), la cual se aplica para generar categorías conceptuales, propiedades y criterios extraídos del discurso infantil.

En particular, se utilizaron los procesos de codificación abierta y axial ${ }^{4}$ y el principio de validez por saturación ${ }^{5}$, tanto para el diseño metodológico como para el análisis del dato cualitativo.

La escogencia de los aportes del MCC o Grounded Theory tiene explicaciones de tipo conceptual y metodológico. La principal razón conceptual se relaciona con el uso de categorías abiertas y axiales, las cuales, pueden ser redefinidas, de acuerdo con el proceso de comparación constante, en varios ejes: el teórico y el pragmático. Adicionalmente, como procedimiento, el MCC posibilita un acercamiento sistemático y comparativo entre los datos y el marco conceptual de apoyo, lo cual lleva al afinamiento de categorías de comprensión durante todo el proceso investigativo. Además, desde una visión sensible al contexto natural de los participantes del estudio (en este caso, el ambiente escolar), se favorece un vínculo más directo con los participantes y una retroalimentación y enriquecimiento compartido.

\subsection{Selección de participantes}

Para esta investigación se trabajó con niñas y niños de diez a doce años, por la adquisición en este momento del desarrollo, de una mayor capacidad de discriminación al recibir información, analizarla y emitir una valoración propia en la cual se refieren emociones morales diferenciadas, al contemplar más posibilidades de reacción emocional ante una situación de castigo.

\footnotetext{
4 La codificación abierta (Open Coding) es aquella parte del análisis consistente en identificar, nombrar, categorizar y describir el fenómeno encontrado en el texto (o cuerpo de datos). La codificación axial es el proceso de relacionar códigos (categorías y propiedades) por una combinación de pensamiento inductivo y deductivo (Glaser y Strauss, 1999; Strauss y Corbin, 1990, cit. en Sánchez, 2007: 49). Esto significa que la codificación abierta y axial es un complemento dentro del proceso de categorización que va desde la identificación hasta la relación y profundización en el análisis.

${ }^{5}$ Al aplicar este principio metodológico el investigador debe analizar, agrupar y estructurar los datos obtenidos en categorías axiales hasta agotar el material, es decir, hasta determinar el límite de contenido de la categoría. (Muchielli, 1996, cit. en Sánchez, 2007: 85). Esto puede implicar crear una nueva categoría si se encuentra que el material residual es consistente con otros hallazgos o bien, desecharlo por no encontrar soporte en otros hallazgos similares.
} 
En la selección de los participantes, se realizó un muestreo no probabilístico de sujetos-tipo, para garantizar la riqueza, profundidad y calidad de la información (Hernández, Fernández y Baptista, 1991) y se siguieron los parámetros cualitativos de selección muestral de Miles y Huberman (1994, cit. en Sánchez, 2007, pp. 52-53). Adicionalmente, se elaboró un consentimiento informado que fue presentado de forma oral y escrita tanto a los participantes como a sus madres y padres. Por medio de este documento se explicaron los objetivos de la investigación, riesgos y beneficios posibles, la duración y participación voluntaria, el retiro voluntario, el manejo de confidencialidad tanto del contenido de las entrevistas como de la identidad de las niñas y los niños.

La muestra total consistió en doce participantes, seis niñas y seis niños con edades entre los diez y los doce años, que cursaban en el momento del estudio el cuarto, quinto y sexto grado. Se entrevistó a cuatro niños por nivel (dos niños y dos niñas), de acuerdo con la definición de criterios de inclusión y exclusión, además de una categorización que se les solicitó a los docentes sobre el comportamiento social (líder, moderado, tímido) de las niñas y los niños recomendados. Dicha caracterización fue realizada a conveniencia según parámetros cualitativos de variación muestral y no en relación con hipótesis previas sobre los posibles resultados. (Véase Sánchez, 2007: 52-53) El Cuadro 1 muestra una síntesis de las características de los participantes:

\section{Cuadro 1}

\section{Participantes del estudio}

\begin{tabular}{ccccc}
\hline Sujetos & Sexo & $\begin{array}{c}\text { Comportamiento social } \\
(\mathbf{L}, \mathbf{M}, \mathbf{T})\end{array}$ & $\begin{array}{c}\text { Edad } \\
\text { Líder }\end{array}$ & $\begin{array}{c}\text { Grado } \\
\text { escolar }\end{array}$ \\
S1 & F & Moderado & 11 & $5^{\circ}$ \\
S2 & F & Moderado & 10 & $4^{\circ}$ \\
S3 & F & Tímida & 12 & $6^{\circ}$ \\
S4 & F & Tímida & 12 & $6^{\circ}$ \\
S5 & F & Tímida & 10 & $4^{\circ}$ \\
S6 & F & Líder & 11 & $5^{\circ}$ \\
S7 & M & Líder & 10 & $4^{\circ}$ \\
S8 & M & Líder & 11 & $6^{\circ}$ \\
S9 & M & Moderado & 11 & $5^{\circ}$ \\
S10 & M & Moderado & 10 & $4^{\circ}$ \\
S11 & M & Tímido & 10 & $4^{\circ}$ \\
S12 & M & & & \\
\hline
\end{tabular}




\subsection{Instrumento}

Para explorar el razonamiento moral emocional infantil sobre las prácticas de castigo parental, se presentaron varios estímulos por medio de secuencias gráficas e historias cortas, con el fin de preguntarles a los participantes sobre lo que hizo y debía hacer el protagonista de las situaciones, sus padres o encargados hipotéticos y el propio entrevistado como madre o padre hipotético, en relación con sus emociones y acciones en una situación paradigmática de conflicto.

Se realizaron tres sesiones individuales de trabajo de campo. El instrumental aplicado se confeccionó y validó para los fines de este estudio; dicho instrumento consta de nueve estímulos diseñados a partir de situaciones hipotéticas de conflicto o situaciones paradigmáticas $^{6}$ en diversos escenarios cotidianos (escolar, familiar y del vecindario) y fueron presentadas como secuencias de láminas e historias hipotéticas, seguidas de algunas preguntas de control, a modo de entrevista semiestructurada. (Para consultar el instrumento elaborado véase Sánchez, 2007, pp. 73, 74, 76)

\subsubsection{Diseño metodológico}

En el proceso de construcción de cada situación paradigmática, y en la elaboración de las preguntas de control ${ }^{7}$ correspondientes, se siguió una estructura similar a la utilizada en estudios revisados sobre la moralidad infantil (Nunner-Winkler y Sodian, 1988; Valtin y Walper, 1996; Leman y Duveen, 1999; Sörbring et al., 2003).

De este modo, la construcción del instrumento consistió en adecuar las situaciones según los siguientes criterios morales, adaptados del estudio de Nunner-Winkler y Sodian (1988):

\footnotetext{
${ }^{6}$ Para este estudio se definió situación paradigmática como aquella que es representativa o ejemplar de una manifestación de la realidad. En este caso, se trata de una situación representativa o ejemplar de eventos que suceden en la realidad social de niños y niñas costarricenses escolarizados, del Área Metropolitana, con características similares a los participantes del estudio. Además, cada situación paradigmática diseñada representa en particular un conflicto o dilema hipotético por resolver, en el contexto familiar, escolar o del vecindario, de modo que el protagonista de cada evento es medular en el conflicto.

${ }^{7}$ Por preguntas de control se entienden aquellas que, como mínimo, es indispensable realizar para la recolección de los datos.
} 
A. El protagonista actúa de forma intencionada ${ }^{8}$, transgrede una norma y provoca un daño intencionado.

B. El protagonista actúa de forma intencionada, transgrede una norma y provoca un daño no intencionado o colateral (no el que esperaba producir).

C. El protagonista no actúa de forma intencionada, pero provoca un daño no intencionado o accidental.

Esto dio como resultado nueve estímulos en total, organizados como se observa en el Cuadro 2:

Cuadro 2

Cantidad de situaciones paradigmáticas según criterios morales

\begin{tabular}{|c|c|c|c|c|}
\hline \multirow{2}{*}{$\begin{array}{l}\text { Situaciones } \\
\text { Paradigmáticas }\end{array}$} & \multicolumn{3}{|c|}{ Criterios morales } & \multirow[t]{2}{*}{ Total } \\
\hline & $A$ & B & C & \\
\hline Láminas & 1 & 1 & 1 & 3 \\
\hline Viñetas & 2 & 2 & 2 & 6 \\
\hline
\end{tabular}

Para las láminas finalmente se obtuvieron tres situaciones paradigmáticas. La situación paradigmática Tipo A ocurre en un contexto escolar y consiste en una disputa entre compañeras o compañeros de escuela; la situación Tipo B se desarrolla en un contexto familiar, con una disputa entre hermanos y hermanas; la situación Tipo C presenta un accidente provocado por amigas y amigos del vecindario mientras se divierten jugando bola.

En el caso de las historias hipotéticas, se realizaron seis versiones, dos historias por criterio. Para el criterio A, la primera historia sucede en un contexto escolar y consiste en una disputa entre compañeras y compañeros de escuela; la segunda historia ocurre en un contexto familiar cuando una niña o un niño por llegar tarde a su casa, incumple con sus deberes escolares y hogareños.

\footnotetext{
${ }^{8}$ Por intención se entiende la actitud deliberada del protagonista de la situación al transgredir una norma y provocar un daño.
} 
Para el criterio $B$, la primera situación en un contexto familiar, trata sobre la destrucción del juguete de una hermana o hermano; la segunda se desarrolla también en el ámbito familiar cuando el protagonista quiebra un adorno de la casa.

Por último, para el criterio $\mathrm{C}$, la primera historia, da cuenta de un niño o una niña que, por descuido, no llega a su casa en el momento acostumbrado, mientras que la segunda historia se relaciona con un niño o una niña que tiene a su cargo a un primo menor y sucede un accidente en el hogar.

En forma paralela a esta elaboración, se definieron las preguntas de control para cada bloque de situaciones (Tipo A, B o C) a modo de entrevistas no estructuradas, pues, como observan Ary y Razavieh, (1997, p. 179), mediante las entrevistas no estructuradas:

Es posible interrogar libremente a los sujetos sobre sus opiniones, actitudes, creencias y otros puntos. Estas entrevistas son flexibles y suelen planearse para adaptarse a los sujetos y a las condiciones dentro de las que se desarrollan. Los sujetos tienen libertad para ir más allá de las simples respuestas y expresar sus puntos de vista en la forma que deseen. Las preguntas pueden desviarse de los planes originales y centrarse en los puntos que parezcan importantes.

\subsubsection{Validación}

Para garantizar la validez de contenido del instrumento, se recurrió a un procedimiento de triangulación cualitativa en dos partes: a) por medio de criterio experto y, posteriormente, b) mediante una prueba piloto con algunos de los participantes del sondeo previo al diseño del protocolo.

El criterio experto estuvo conformado por tres evaluadores, profesionales en Psicología: una especialista en clínica infantil, un investigador y docente de la Escuela de Psicología de la Universidad de Costa Rica que ha trabajado el tema de la subjetividad infantil, y una docente de la Escuela de Psicología de la Universidad de Costa Rica del área del desarrollo humano infantil. Por medio de un cuestionario diseñado con una escala Likert se midió la validez de constructo, considerando los siguientes aspectos del instrumento:

- La estructura formal de las viñetas en cuanto a diseño, redacción y ortografía.

- El lenguaje utilizado (adecuado al contexto de la población del estudio y a sus participantes).

- La correspondencia entre cada situación paradigmática y el criterio al que pretendía responder. 
La evaluación del cuestionario se hizo de forma cualitativa, de acuerdo con una categorización de las observaciones y las calificaciones de los evaluadores.

Seguidamente, se realizó una prueba piloto con algunas niñas y niños entrevistados en sondeos previos y se les presentó el instrumental diseñado para corroborar si cumplía con el propósito de explorar su concepción sobre las prácticas de castigo parental. En esta etapa, la validación consistió en comprobar: a) la claridad de las instrucciones y consignas; b) el planteamiento de las preguntas de control para cada situación paradigmática; c) la correspondencia entre las preguntas de control, su respectiva situación paradigmática y el criterio correspondiente.

Luego de esta fase de validación, se afinaron algunas de las situaciones paradigmáticas y se eliminaron las inconsistencias encontradas en la confección de las historias presentadas. Dichos afinamientos consistieron en eliminar anidamiento de ideas en la redacción de las historias, incorporación de otros escenarios pues privaba el entorno familiar, incorporación de situaciones de conflicto por incumplimiento de deberes delegados pues según los expertos esto era una típica situación conflictiva en la cotidianidad infantil costarricense. También se revisaron posibles vicios de sexismo para evitar inducir estereotipos y prejuicios de género.

\section{Resultados}

\subsection{Las prácticas de castigo parental desde la perspectiva infantil}

En términos generales, en las valoraciones de los participantes se encontró un acuerdo social en el tipo de medidas aplicadas como castigo ante la creencia de que se cometió una falta por un comportamiento inadecuado.

Para los entrevistados, los castigos pueden ser adecuados o no pero son necesarios. Los más comentados se relacionan con la privación de privilegios y pueden ir acompañados de amonestación verbal, sermón o explicaciones dialógicas o inductivas.

Las prácticas de castigo mencionadas con más frecuencia son las que se aplican de forma directa e inmediata, vinculadas a la falta cometida o al resultado de la acción y en relación con la personalidad del niño o la niña. Suelen ir acompañadas del sermón y se utilizan para que las hijas e hijos: "piensen en la acción que hicieron", "que me haga reflexionar". Otra alternativa al castigo es el diálogo o "hablar" como un recurso previo que puede provocar el mismo resultado y evitar la aplicación de un castigo. 
Adicionalmente, la restricción de actividades predilectas como jugar bola, ver televisión, jugar con la computadora, con videojuegos y salir con las amigas o amigos también se expresan como prácticas de castigo frecuentes. También, se da la privación de otros privilegios como el retiro de la mesada para reponer un objeto dañado, no comprar juguetes u otros objetos de consumo o no salir a pasear. Otros castigos pueden ser de restricción física, como el encierro en el cuarto, no salir a jugar durante un tiempo o la prescripción de actividades alternativas, como estudiar en vez de jugar.

Una síntesis de los resultados obtenidos puede observarse en el Cuadro 3:

\section{Cuadro 3}

Resultados obtenidos según situaciones paradigmáticas

\begin{tabular}{|c|c|c|c|}
\hline $\begin{array}{l}\text { Contexto } \\
\text { situacional }\end{array}$ & A & B & C \\
\hline Entre pares & $\begin{array}{l}\text { Disculpa, perdón } \\
\left(\neq q \delta^{\lambda}\right)\end{array}$ & $\begin{array}{l}\text { Disculpa, perdón }(\neq q \text { ठ) } \\
\text { Atender al afectado(a) }\end{array}$ & Soluciones colectivas \\
\hline $\begin{array}{c}\text { Escuela } \\
\text { (maestra, } \\
\text { director) }\end{array}$ & $\begin{array}{l}\text { Suspensión, } \\
\text { expulsión }^{9} \\
\text { Sermón }\end{array}$ & $\begin{array}{l}\text { Suspensión, expulsión } \\
\text { Sermón }\end{array}$ & No aplica \\
\hline Familia & $\begin{array}{l}\text { Sermón } \\
\text { Privación de } \\
\text { privilegios (no ver } \\
\text { TV, no salir, no } \\
\text { jugar bola, } \\
\text { videojuegos, } \\
\text { computadora) } \\
\text { Castigo corporal } \\
\text { Diálogo y } \\
\text { consejería }\end{array}$ & $\begin{array}{l}\text { Sermón } \\
\text { Privación de privilegios (no ver } \\
\text { TV, no salir, no jugar bola, } \\
\text { videojuegos, computadora) } \\
\text { Castigo corporal } \\
\text { Diálogo y consejería }\end{array}$ & $\begin{array}{l}\text { Diálogo- consejería } \\
\text { disculpa } \\
\text { Alternativas } \\
\text { preventivas (Jugar en } \\
\text { el patio o en el } \\
\text { parque) } \\
\text { Privación de } \\
\text { privilegios (reponer el } \\
\text { objeto, no salir) }\end{array}$ \\
\hline & $\begin{array}{l}\text { Estrategias } \\
\text { combinadas }\end{array}$ & Estrategias combinadas & $\begin{array}{l}\text { Estrategias } \\
\text { combinadas }\end{array}$ \\
\hline
\end{tabular}

${ }^{9}$ Esta mención de expulsión se respeta pues provino del discurso infantil, no obstante, según la normativa educativa costarricense para las escuelas públicas debe entenderse como sinónimo de suspensión. Volumen 9, Número 2, Año 2009, ISSN 1409-4703 
Como puede observarse en el cuadro, para las niñas y los niños entrevistados, el castigo parental operó como una alternativa posible, mas no la única ni predilecta cuando se valoró que el protagonista de las historias cometía una falta y ocasionaba un daño evidente, debido a un comportamiento inapropiado de su parte. Para los participantes, el castigo puede ser temido y no gustado, pero necesario; sin embargo, no lo refieren como la única opción para regular el comportamiento infantil.

Los castigos más importantes, severos y directos son referidos cuando valoraron que la gravedad de la falta cometida y el daño ocasionado: "lo expulsaron tal vez... porque hacía bromas pesadas y agredía y todo". Esto está relacionado con su comprensión de la intención como actos deliberados que implican transgredir una norma y provocar un daño. Para ellas y ellos, tales actos son más sancionables que los actos no intencionados o los accidentes. No obstante, los accidentes conllevan el riesgo de producir daños a terceros o a sí mismos y, según la gravedad de las consecuencias, amerita tomar medidas correctivas o preventivas que pueden o no implicar el uso de castigos: "la próxima vez los llevaría al parque o algo así".

De esta forma, también los entrevistados aplicaron castigo cuando se ocasionó un daño, aun cuando el protagonista no lo hiciera de forma intencionada: "lo hicieron sin culpa. Estaban jugando bola tranquilos". Sin embargo, por ejemplo, se aplicó como castigo el encierro en el cuarto: “... si uno está jugando cerca de la casa y tiene vidrios, es mejor jugar suavecito y no patearla tan duro"; en este caso, la función que se atribuye al castigo es: "para que aprendan la lección y sepan que las cosas cuestan dinero".

Ahora bien, respecto de la comprensión del castigo parental, en el caso de las niñas se encontró una moralidad más orientada hacia el cuidado y la responsabilidad sobre la conducta moral-emocional, hallazgo encontrado por Gilligan (1982) en sus estudios sobre la moralidad femenina. La tendencia mayor en las niñas fue a valorar la situación en términos del vínculo interpersonal y la afectividad: "le hubiera hablado para ver si reflexionaba y la hubiera acompañado más para ver si cambiaba”, "uno no castiga por no querer".

Las niñas, en mayor medida, recurren a una combinación de sentido desde la moralresponsabilidad-cuidado hacia la consecuente decisión adecuada o justa. Fueron más empáticas. Además, refirieron más explicaciones a la hora de justificar los castigos para producir mayor aceptación de sentimientos negativos, "decirle que eso no se hacía porque seguro a ella no le hubiera gustado que hubiera sido ella y que la hubieran molestado... pudieron haberla castigado y que le pidiera disculpas a Lucía". También estuvieron más 
dispuestas a retirar un castigo si se consideraba que el protagonista se retractó y se disculpó.

En el caso de los niños, la moralidad se orientó en mayor medida hacia lo correcto y lo incorrecto, de acuerdo con su concepción de intención deliberada y justicia, por la acción en sí y el resultado: "eso no se hace (hacer bromas pesadas)... así no hay que ser con las personas". Los niños dieron más importancia a la valoración sobre la gravedad de las consecuencias de las acciones y esto derivó en que los castigos aplicados por ellos fueron más severos.

No obstante, estas tendencias encontradas no fueron exclusivas por condición de género, en otras palabras, algunos niños mostraron una actitud más empática que otros y también algunas niñas orientaron su discurso en términos de su concepción de justicia y deber moral.

\subsection{Castigo corporal y prácticas de maltrato}

En cinco de los casos (cuatro niños y una niña) se menciona el uso del castigo corporal como medio de corrección o como medida necesaria por reincidencia de lo que se considera un comportamiento inadecuado (previa advertencia de las madres y los padres). Detrás de la prescripción de castigos más severos, se ve reflejado en el discurso infantil la introyección de un mensaje de poder centrado en la figura parental y que tiene que ver con una amenaza que pretende controlar el comportamiento: "ya se lo habían advertido", "para que aprenda".

Para uno de los niños entrevistados, el castigo corporal se menciona como algo necesario para corregir a las hijas e hijos siempre que no pase un límite: "castigar adecuadamente es no dejarlos todos rojos y así". Es decir, si se percibe una función educativa o correctiva del castigo corporal se le considera necesario aunque no guste y lastime la emocionalidad infantil, siempre y cuando se aplique "de forma moderada".

Estos hallazgos corroboran las tesis de Campos y Mejía (2001) y Strauss (1994) sobre la aceptación del uso de la fuerza física contra las niñas y los niños al percibir esta función educativa, haciendo del castigo corporal una práctica legitimada socialmente, aún cuando sea violenta. En este caso particular, la aceptación del castigo corporal se produce cuando se internaliza la creencia de que se aplica "por su propio bien" o "por merecimiento".

En el caso de este niño, la aceptación del castigo corporal se produjo por una creencia relevante con contenido ideológico-religioso: "no es tampoco pegarles demasiado, dejarlos sangrientos, pero en la Biblia dice que hay que pegarle a los hijos no de mal modo pero Volumen 9, Número 2, Año 2009, ISSN 1409-4703 
depende de cómo los padres lo pongan en práctica". Para él el castigo corporal se justifica porque se trata de "pegarles adecuadamente, hablar con ellos; castigar adecuadamente es no dejarlos todos rojos y así". Y en este caso sí se considera efectivo: "con eso aprenden sí porque hay que corregirlos", aunque tenga consecuencias negativas en la niña o niño: "a los hijos les duele pero a la misma vez se sienten que los están corrigiendo les están haciendo un bien".

En este discurso se refleja el planteamiento de Alice Miller (1998) sobre la validez universal de las prácticas maltratantes hacia las niñas y los niños, pues se acepta el castigo corporal "por el bien del niño" como mecanismo, de lo que la autora llama la pedagogía negra. Es decir, no se cuestiona una práctica maltratante pretendidamente educativa.

Con excepción de este caso, hubo inconformidad de la mayoría de los participantes hacia el uso del castigo corporal: "porque a nosotros nos duele", "porque lastima sentimientos", "uy mi papá no me quiere porque me pega a mí", "cuando llegan los papás y les pegan... medio tristes porque piensan que los están agrediendo"; y dejaron entrever en su discurso la inefectividad del castigo corporal: "que les peguen porque no se arregla nada con golpes", "no sirven porque los hijos se enojan... se vengan de los papás y lo vuelven a hacer", ya que, según sus opiniones siempre era mejor usar otras alternativas planteadas como soluciones para evitar recurrir al maltrato: "los hijos con los castigos se sienten preocupados, asustados, con castigos bajitos lo pueden volver a hacer pero con el tiempo cambian", "mejor hablar, castigos no muy fuertes", "con lo que más le gusta, tal vez así les duele hacer lo que hicieron... si a uno le gusta mucho ver tele".

De acuerdo con estos principios aportados por las niñas y los niños entrevistados, se desprende cómo en términos de la relación madres/padres-hijas e hijos, el factor castigo corporal tiene implicaciones negativas en la relación de apego; se produce un aprendizaje pero por distanciamiento, ya que se trata de un estímulo aversivo, como tal produce rechazo y malestar emocional, lo cual tiene implicaciones negativas en el desarrollo de la personalidad, tal y como lo plantean Strauss (1994) y Miller (1998) al haber estudiado los efectos duraderos negativos del castigo corporal.

Para los participantes, los castigos menos adecuados son el maltrato físico, el castigo corporal, la restricción física como el encierro en el cuarto y la privación de actividades muy importantes para el niño o la niña según sus preferencias; como consecuencias negativas se refiere que son menos adecuados o "peores" porque "lastiman sentimientos"o también porque la propia experiencia les enseña que eso no es lo mejor: "porque a mí no me gusta, 
entonces no lo haría" y con esto expresan un sentido de respeto hacia su propia emocionalidad e integridad y la relación con los demás.

Otros castigos valorados como "peores" son la restricción de actividades sociales muy importantes para el niño o la niña, por ejemplo, salir con los amigos o las amigas o actividades escolares que implican reconocimiento social, como participar de la escolta. Adicionalmente, consideran que los castigos que se aplican de forma indirecta, es decir, desvinculados de una explicación y probablemente de forma arbitraria, también provocan consecuencias emocionales negativas en los hijos y las hijas: "quitarles algo y no volvérselos a dar nunca más porque ellos no saben por qué se los quitaron... (respecto a los sentimientos agrega) mal porque creen que el papá es malo porque le quitan algo que no saben por qué es".

\subsection{Castigos más adecuados y efectivos}

Para las niñas y los niños de este estudio, los castigos más efectivos son aquellos que permiten el aprendizaje y la reflexión sobre las consecuencias de las acciones: "el niño se siente muy mal cuando le pegan mejor regaño o no ver tele o no salir. Cuando los papás pegan es porque el hijo hace algo malo, golpean a alguien o regañan o le dice algo, pero no es bueno... con los buenos castigos los hijos pueden aprender" y son más aceptados, pues como "buenos castigos" no dañan la integridad emocional, física, intelectual o social de las hijas y los hijos. De ahí la importancia de las medidas disciplinarias como medios educativos. Para ello los participantes recomiendan el uso del diálogo o de explicaciones que puedan promover el aprendizaje sobre lo ocurrido, en el sentido de un desarrollo personal.

Lo anterior explica la propuesta infantil de otro tipo de medidas que pueden acompañar o sustituir el uso de castigos. En este sentido, algunos participantes refieren que no se debe castigar cuando ocurre una falta por primera vez, no obstante, advierten utilizar el castigo si vuelve a suceder. En otros casos, el castigo no se asigna, sino que se opta por el diálogo, que el padre o la madre hablen con su hija o hijo y le hagan ver la equivocación cometida.

Desde esta postura personal, las técnicas inductivas, como el diálogo o la explicación, son deseadas como medio educativo en el sentido de que para los participantes es preferible "arreglar las cosas" dialogando, hablando; sin embargo, a la hora de proponer soluciones a una situación de conflicto, no siempre se convierte en la primera opción por seguir o en la única. Lo que más se advierte en las expresiones de las niñas y los niños entrevistados es la transmisión de la propia experiencia con relación a los castigos recibidos, visto como Volumen 9, Número 2, Año 2009, ISSN 1409-4703 
necesario aunque no sea lo más deseado. Es mediante una reflexión posterior, al asumir un rol como padre o madre hipotéticos, cuando esta opción puede sobresalir; es decir, al repensar qué sería lo mejor para resolver una situación conflictiva, en algunos casos se corrigen las medidas de castigo y se considera hablar como lo más importante, haciendo ver las consecuencias de las acciones para sí mismo y para los demás.

Al hablar de los castigos, la intención de las madres o padres fue percibida como los motivos por los cuales es necesario aplicar ese tipo de medida disciplinaria. Esta intención se justificó desde una atribución moral emocional sobre un comportamiento, y por creencias relevantes sobre lo deseable o correcto. Esto tiene una relación directa con la intención que se percibe del protagonista acerca de sus deseos, sentimientos y creencias, así como el juicio hecho sobre sus actos: "se lo merecía... por la mala actuación que tuvo", "eso no se hace", "por irresponsable", "es así porque así lo educaron"; como consecuencia, se señala que los castigos se aplican por estos comportamientos inadecuados, por merecimiento o para brindar apoyo y orientación. Esto, a su vez, se relaciona con lo que para ellas y ellos sería la funcionalidad de las prácticas de castigo: educar y corregir las acciones que no estuvieron de acuerdo con lo esperado por las madres y los padres: "se tiene que controlar un poco", "para que aprenda que debe avisar, que si va a algún lado, para ellos saber dónde está y no se preocupen tanto".

\section{Discusión}

\subsection{Teorías infantiles sobre las prácticas de castigo parental. Enfoque moral- emocional}

En este estudio la definición de teorías infantiles sobre las prácticas de castigo parental resulta compleja; se trata de la comprensión de las niñas y los niños entrevistados sobre su realidad de crianza, ejemplificada de múltiples maneras por medio de prácticas, creencias, valores, expectativas, principios y criterios aportados desde la riqueza del sentido común, el pensar-sentir de las cosas y los fenómenos más inmediatos desde el espejo de sí mismo. Cada niña o niño es su propio espejo, reflejo de su historia. Su pensamiento deja entrever cómo ha calado ésta, y se expresa ahora y hacia el futuro: "digamos, usted me va a regañar o algo así... digamos que no le prestarían nada a uno porque uno agarra algunas de las veces sin permiso", "no quitarle algunas otras cosas, pero no dejarlo salir, no dejarlo salir al 
frente ni nada...bueno por un tiempo...hasta que él reflexionara. Sería el mejor castigo", "esas son las cosas que tal vez ellos le enseñaron a ella que no debían hacer".

En los discursos infantiles pueden encontrarse las palabras de los otros, las de las madres y los padres, de los hermanos, compañeros, amigos, vecinos y maestros; lo que convirtió el razonamiento de estas niñas y niños en un proceso no unidireccional sino interconectado; en otras palabras, vincular y vinculante: "se sintió bien porque los papás cuesta mucho que se comuniquen con los hijos". Estas reflexiones infantiles, desde una teoría del sentido común, muestran una amplia percepción sobre la justicia, lo correcto y lo incorrecto con base en un dilema moral emocional, expresando una ética particular basada en la empatía "porque al sentirse regañado y si él vuelve a quebrar algo no le vuelven a dar nada, digamos no le compran nada", "se sintió mal porque a uno también le gusta jugar, pero primero está el estudio".

La manera como son asumidas y explicadas las prácticas de castigo parental por las niñas y los niños del estudio permitió acceder a nuevas comprensiones y evoluciones que dimensionan la crianza como un fenómeno continuo, complejo y dinámico. La teoría infantil, en este caso, parte de una postura personal que a la vez es resultado del vínculo primario con las madres y los padres, y este vínculo es medular como motor de unas $u$ otras formas en que el desarrollo moral emocional se ve expresado desde el pensamiento "los papás castigan cuando tienen un motivo, para que aprenda la lección... porque así los niños aprenden que eso no se hace y ya saben y no lo tienen que volver a castigar", "palabras necias oídos sordos, lo hubiera castigado porque está mal hecho... no ver televisión, no salir a jugar".

Con esto se explica que la aplicación de un castigo es aceptable para las niñas y los niños en cuanto reconozcan una función socializadora; en particular, se encontró que el ser bien portado, obediente y respetuoso de las reglas de la familia y de la sociedad es el modelo de sujeto que se espera producir: "obedecer a los papás", "si uno sabe lo que tiene que hacer que lo haga", "tal vez no muy respetuoso, pero respetuoso. Y comportándose bien". En este sentido, se corrobora la tendencia planteada en el estudio de parentaje (Keller et. al, 2005) sobre la deseabilidad social y el colectivismo como metas de socialización infantil en la sociedad costarricense: "así no hay que ser con las personas", "está teniendo como una actitud mala". En el vínculo de apego hacia los padres y las madres, el castigo parental tiene, entonces, una función cultural y de desarrollo personal. 
No obstante, de acuerdo con los hallazgos obtenidos a partir del razonamiento de los participantes, el tema de la aceptación de los castigos presenta dos dimensiones en conflicto: por una parte, se produce su aceptación por la internalización del discurso parental, más desde lo cognitivo "para que aprendan", "por hacer ese error"; pero, por la otra, se vive con malestar y rechazo, "a nadie le gusta que lo castiguen", más desde lo emocional. Este malestar emocional, desde lo afectivo, permite dar cuenta de la necesidad de las niñas y los niños de que existan otras medidas para solucionar los conflictos, en concreto, el diálogo y las explicaciones sobre cómo su comportamiento afecta a los demás.

Siguiendo esta línea, en la comprensión infantil de las situaciones presentadas, las explicaciones sobre el propio comportamiento y el de los demás en situaciones de conflicto demostraron una relación recíproca entre afectos y cogniciones, puesto que el razonamiento infantil estuvo sustentado por emociones y creencias atribuidas a los implicados en la situación paradigmática: "creo que los padres quieren mucho a sus hijos y los quieren mucho y a ellos les duele lo que les pase o algo así... también se sacrifican", "cuando yo estoy peleando así con mi primo o algo, y yo le pego, yo siento que, que, es que yo no le quiero pegar; bueno, yo le pego, y entonces es como que me estoy pegando a mí mismo o así".

En estos ejemplos, puede observarse que ante la identificación y atribución de una emoción de malestar por la situación, se produce un cambio de registro que da pie a un juicio moral; es decir, las explicaciones tienen un trasfondo afectivo. Esta interacción entre emociones y cogniciones estuvo presente a lo largo de las respuestas de las niñas y los niños. Por ejemplo, cuando se dice que el protagonista se sentía culpable luego se plantea que el niño o la niña reflexiona y pide disculpas. Si es objeto de un castigo como la restricción de alguna actividad que le guste hasta que piense en la acción que hizo, entonces esto puede implicar un malestar emocional por el castigo recibido a la vez que un nuevo cambio de registro emocional hacia un mayor bienestar porque se logró un aprendizaje "mejor porque están aprendiendo la lección".

En relación con los castigos, las prácticas correctivas, educativas, de amonestación, se perciben aversivas en muchos casos para los participantes, pero, a la vez, se producen en medio de una relación de apego. Por esto, cuando se atribuyen sentimientos, creencias o valores a los protagonistas y a sus madres y padres en las situaciones paradigmáticas, se producen muchos paralelismos y correspondencias. En concreto, tanto unos y otros sienten malestar ante las situaciones que implican introducir castigos; este malestar se expresa como enojo, preocupación, miedo o susto, tristeza y decepción; en este sentido, las 
emociones son vinculantes y el castigo se introduce como una forma de reorganizar la subjetividad. Desde la concepción de los participantes, se desprende que el castigo no es sólo para las niñas y los niños, sino, también, para las madres y los padres, aun cuando sea una decisión parental. Esto se ve reflejado en las reflexiones infantiles, cuando expresaron que a las madres y los padres no les gusta castigar, pero lo hacen porque lo consideran necesario y esto les causa dolor, igualmente esto causa malestar en las hijas y los hijos.

A partir de los hallazgos obtenidos, la reflexión de las niñas y los niños participantes de este estudio sobre las prácticas de castigo parental más adecuadas, está determinada por una serie de factores interrelacionados que se deben tomar en consideración:

- La indagación sobre lo acontecido.

- La valoración sobre la intención percibida de provocar un daño; es decir, si se consideró que fue a propósito (con culpa) o no.

- La identificación del tipo de infracción cometida o comportamiento inapropiado.

- El tipo de daño ocasionado.

- La frecuencia de aparición del comportamiento inadecuado y si es primerizo o reincidente.

- La atribución sobre la personalidad del niño, rasgos de comportamiento y carácter, gustos y preferencias.

- La atribución sobre la personalidad de las madres y los padres, y su estado anímico en el momento de la situación.

- La expectativa de efectividad del castigo que se asigne.

Adicionalmente, se reconoce en este estudio que el aprendizaje sobre las prácticas de castigo parental fue posible para los participantes, de acuerdo con varios fenómenos que se dieron de forma paralela:

- Una toma de rol mediante una capacidad empática que posibilitó abstraer sobre la condición particular del otro, en algunos casos por identificación con la propia experiencia, donde se presentó una confluencia entre el sentido de sí mismo y el sentido de los demás.

-A lo interno de la situación se aprende por ensayo y error; ante una emoción negativa que provoca malestar personal, se activan esquemas cognitivos, los cuales permiten extraer una comprensión de lo sucedido. En este sentido, el desarrollo moral emocional 
se sostiene en ese proceso dinámico, de comparación entre la realidad objetiva para el niño o la niña, sus reacciones emocionales y cogniciones ante una situación dada y la interrelación entre estos elementos.

- La capacidad empática comprende tanto aspectos emocionales como cognitivos y morales, pues desde el lugar del otro es posible hipotetizar sobre sus sentimientos, rasgos de comportamiento, creencias, deseos y acciones. Si bien esta cualidad no es consciente para los participantes, se produce como resultado de responder ante situaciones donde se presentan dilemas morales y a partir de allí se genera la necesidad de aportar soluciones según los recursos personales disponibles. Lo anterior se ve reflejado en la síntesis de lo aprendido que hacen los participantes al final de las tres sesiones del trabajo de campo:

"que no tengo que hacer cosas malas ni nada así, porque me castigan o algo así".

"algunas veces se cometen errores, pero después se resuelven...se corrigen.

"a ser obediente y no ser desobediente".

"errores que cometen los chiquitos para que nosotros no podamos cometerlos".

"a los hijos no hay que maltratarlos, que... los castigos son para que ellos aprendan las lecciones".

"tener más cuidado, tratar de no pelear, ser responsable con las cosas".

En estas enseñanzas personales, los participantes reflejan su propia ética, una integración para la vida, desde el sentido común, desde lo social-personal aprendido y nos acercan a la comprensión de las prácticas de castigo parental como factores medulares en el desarrollo personal, entendido como un proceso complejo y dinámico que orienta el aprendizaje.

Lo anterior también se evidencia cuando los participantes asumieron el lugar hipotético del adulto pues expresaron como resultado: "aprendo cómo educar a los hijos cuando uno sea grande", "los padres se sienten mal porque ellos nos quieren", "si me castigan, me siento mal, entonces no me gustaría castigar a mis hijos ni pegarles, a uno no le gusta que le hagan eso", "si no aprenden por la buena tienen que aprender así castigándolos". En estas expresiones los niños y las niñas dejan ver la influencia de sus padres, madres o cuidadores, pero también el arraigo de creencias y expectativas que posiblemente serán llevadas a la práctica en la vida futura.

Volumen 9, Número 2, Año 2009, ISSN 1409-4703 
De esta forma, la toma de rol como padre o madre hipotéticos permitió visualizar varias posiciones que evidencian la dinámica del proceso reflexivo de los niños y las niñas:

- Confirmar las medidas atribuidas a las madres y los padres del protagonista, argumentando que harían lo mismo que ellas y ellos hubieran hecho.

- Revalorizar y mitigar las medidas atribuidas a las madres y los padres del protagonista en intensidad o duración, planteando que el castigo sería menor o que no se castigaría y solamente se hablaría con el protagonista.

- Aumentar las sanciones atribuidas a las madres y los padres del protagonista; esto, cuando se consideró que el daño fue intencionado y grave.

Lo anterior implica diferentes niveles de reflexión; la niña o el niño revaloriza su posición frente a los adultos y esto es posible mediante una comparación entre su realidad cotidiana, lo que para Wellman (1995) sería la realidad física, y la propia asimilación y explicación de esas experiencias, o el concepto de realidad mental según este mismo autor. En este sentido, la moralidad infantil sobre lo correcto y lo incorrecto se refleja desde varios lugares de sí mismo, por medio de una toma de rol empática: a) desde el lugar de hija o hijo; b) desde el lugar del coetáneo que fue visto como el amigo(a), el cómplice o la víctima; c) desde el lugar de las madres y los padres de los protagonistas de las situaciones paradigmáticas, los cuales fueron vistos como afectados, como figuras de autoridad o de protección y apoyo, d) desde el lugar del padre o la madre hipotéticos.

Para otros participantes esta posición resultó difícil: "es que no entiendo, soy muy chiquitito", "no sé cómo se sentía", "iqué van a sentir!" y los aportes infantiles se hicieron más desde su propio lugar de niña o niño: "no sé, yo soy el hijo", expresando fielmente su postura personal actual.

\section{Consideraciones finales}

- El estudio de la teoría infantil sobre las prácticas de castigo parental, permite conocer la visión global que tienen las niñas y los niños sobre lo sucedido en una situación de crianza, tomando en cuenta el contexto de las acciones y los principales actores del evento ocurrido. Es por ello que antes de la aplicación de un castigo se hace un reconocimiento de intenciones y de otro tipo de medidas, como por ejemplo, la disculpa entre hermanas y hermanos, compañeras y compañeros y posteriormente se valora si 
aún es necesaria la aplicación de un castigo. No obstante, se encontró también, a partir del razonamiento infantil, que la utilización de castigos deja de lado, en la mayoría de los casos, la comprensión y solución alternativa que pueden aportar las niñas y los niños pues normalmente no se facilita una actitud de diálogo y concertación con las madres y los padres.

- Este reconocimiento de la importancia que otorgan los participantes al diálogo y la concertación en situaciones conflictivas tiene implicaciones para la educación y la crianza en términos de poder recurrir a estrategias inductivas, de mayor participación y propositivas, en un enfoque de comunicación no violenta para la resolución de conflictos, tanto en el ámbito familiar como en el educativo y comunitario.

- Asimismo, el proporcionar espacios de diálogo, mediante metodologías constructivistas, como la utilizada en este estudio, posibilita la emergencia de nuevas alternativas y procesos mentales cada vez más conscientes en las niñas y los niños, convirtiéndose en un motor para el esclarecimiento de situaciones complejas, puesto que se propicia, gracias a recursos creativos y didácticos, la imaginación infantil, la proactividad y el interés en situaciones cotidianas que frecuentemente son invisibilizadas.

Por otra parte, como principales alcances del instrumento utilizado se encontraron los siguientes:

- Las situaciones paradigmáticas funcionan como representaciones ejemplares de eventos de la vida cotidiana de las niñas y los niños entrevistados produciendo interés y aprendizaje personal al realizar inferencias cognitivas y morales.

- La utilización de historias hipotéticas permite a los participantes inferir juicios y emociones a partir de situaciones paradigmáticas, resulta un instrumento atractivo y lúdico; adicionalmente, produjo identificación con sus historias de vida, lo cual enriqueció la información obtenida.

- Usar bloques de historias y mayor cantidad de estímulos produce gran riqueza de datos. El resultado es contar con suficiente información para un análisis multidimensional, que permita la elaboración necesaria para la definición de categorías abiertas y axiales de sentido. Además se propicia un acercamiento más sensible a la realidad, no lineal ni unilateral. 
- La metodología diseñada puede ser utilizada para temas afines al explorado en este estudio. Las láminas e historias pueden ser utilizadas con diversas aplicaciones (de investigación, clínicas o educativas, principalmente), fueron producidas localmente y validadas por profesionales expertos para población infantil costarricense, urbana, de clase media y escolarizada.

- Otros temas de investigación que pueden ser explorados desde la perspectiva infantil mediante la metodología diseñada serían: relación de pares, amistad, relación entre hermanas y hermanos, violencia en las escuelas, resolución de conflictos en ámbitos de la cotidianidad de las niñas y los niños. Además se hace necesario incorporar la consideración del género y del comportamiento social infantil, aspectos que en este estudio no constituyeron el énfasis principal.

\section{Referencias}

Anguera, María T., Arnau, Jaume, Ato, M., Martínez, Rafael, Pascual, J., Vallejo, Guillermo. (1998). Metodología de las ciencias del comportamiento. Madrid: Síntesis Psicológica.

Ary, Donald, Cheser Jacobs, Lucy, Razavieh, Ashgar. (1997). Introducción a la Investigación Pedagógica. Segunda edición. México D.F: Mc Graw-Hill.

Bowlby, John. (1989). Una base segura. Aplicaciones clínicas de la teoría del apego. Buenos Aires: Paidós.

Bowlby, John. (1999). Vínculos afectivos: Formación, desarrollo y pérdida. Madrid: Ediciones Morata.

Campos, Domingo. (2000). El mundo de los niños y el mundo de la televisión. El desarrollo de las teorías infantiles sobre el mundo social. San José: Instituto de Investigaciones Psicológicas, Universidad de Costa Rica.

Campos, Domingo. (2001a). El mundo infantil y el mundo de la economía. El desarrollo de las teorías infantiles sobre la economía. San José: Instituto de Investigaciones Psicológicas, Universidad de Costa Rica.

Campos, Domingo. (2001b). El mundo infantil y el mundo de la política. El desarrollo de las teorías infantiles sobre la política. San José: Instituto de Investigaciones Psicológicas, Universidad de Costa Rica.

Campos, Arliany y Mejía, Tatiana. (2001). El Maltrato Físico Infantil: la percepción de los niños y las niñas. Tesis para optar por el grado de Licenciatura en Psicología, Escuela de Psicología, Universidad de Costa Rica, San José, Costa Rica. 
Defensoría de los Habitantes de la República de Costa Rica. (2007). Proyecto de Ley Abolición del Castigo Corporal contra las personas menores de edad. Recuperado el 20 de junio de 2007 del sitio web http://www.dhr.go.cr/nopeguemos/pdf/proyecto.pdf.

Dobles, Ignacio. (1994). El estudio psicológico del desarrollo moral: consideraciones teóricas y metateóricas. Iztapalapa, 35, 21-54.

Eisenberg, Nancy, Spinrad, Tracy, Losoya, Sandra H., Fabes, Richard A., et al. (1999). The relations of parental affect and encouragement to children's moral emotions and behavior. Journal of Moral Education, 28 (3), 323-338.

Eisenberg, Nancy, Shea, C., Carlo, G. y Knight, G. P. (1991). Empathy-Related Responding and Cognition: A "Chicken and the Egg" Dilemma. En W. M. Kurtines y J. L. Gewirtz (eds.), Handbook of moral behavior and development, 2: Research (pp. 63-88). New Jersey: Lawrence Erlbaum Associates.

Evans, Ian M, Galyer, Karma T y Smith, Kyle J. H. (2001). Children's Perceptions of Unfair Reward and Punishment. The Journal of Genetic Psychology, 162 (2), 212-227.

Fundación Save The Children. (s.f.). Poniendo fin al castigo físico contra la niñez. Cómo hacerlo posible [CD-ROM]. Suecia: Save The Children.

Gilligan, Carol. (1982). In a different voice: Psychological theory and women's development. Cambridge: Harvard University Press.

Glaser, Barney y Strauss, Anselm. (1999). The Discovery of Grounded Theory: strategies for qualitative research. New York: Aldine de Gruyter.

Keller, Heidi, Kuensemueller, Petra, Abels, Monika, Voelker, Susanne, Yovski, Relindis, Jensen, Henning, Papaligoura, Zaira, Lohaus, Arnold, Rosabal-Coto, Mariano, Kulks, Daniela y Mohite, Prerna. (2005). Parenting, Culture and Development. A Com parative Study. San José: Instituto de Investigaciones Psicológicas, Universidad de Costa Rica.

Kohlberg, Lawrence. (1967/1969). Stage and Sequence: The Cognitive-Developmental Approach to Socialization. En D. A. Goslin (Ed.), Handbook of socialization theory and research (pp. 347-480). Chicago: Rand Mc Nally y Company.

Leman, Patrick J. y Duveen, Gerard. (1999). Representations of authority and children's moral reasoning. European Journal of Social Psychology, 29, 557-575.

León, Ana. (2001). Los retos que enfrenta la familia de cara al nuevo siglo, desde la perspectiva de los niños y las niñas. En I. Vega y A. Cordero (eds.), Realidad familiar en Costa Rica (pp. 59-84). San José: Instituto de Investigaciones Psicológicas. Universidad de Costa Rica.

Miles, Matthew y Huberman, A. Michael. (1994). Qualitative data analysis: an expanded sourcebook. London: Sage Publications. 
Miller, Allice. (1998). Por tu propio bien. Raíces de la violencia en la educación del niño. Barcelona: Tusquets Editores.

Miller, Allice. (2002). La madurez de Eva. Una interpretación de la ceguera emocional. Barcelona: Paidós.

Mucchielli, Alex. (1996). Diccionario de métodos cualitativos en ciencias humanas y sociales. Madrid: Editorial Síntesis, S.A.

Nunner-Winkler, Gertrud y Sodian, Beate. (1988). Children's Understanding of Moral Emotions. Child Development, 59, 1323-1338.

Packer, Martin y Richardson, Ellen. (1991). Analytic Hermeneutics an the Study of Morality in Action. En W. M. Kurtines y J. L. Gewirtz (eds.), Handbook of moral behavior and development, 1: Theory (pp. 335-371). New Jersey: Lawrence Erlbaum Associates.

Papalia, Diana, Olds, Sally y Feldman, Ruth. (2005). Desarrollo humano. México D.F.: Mc Graw-Hill.

Patronato Nacional de la Infancia, Oficina de Planificación, SII-PANI. (2003, I trimestre). Cantidad de motivos de atención a niños, niñas y adolescentes según Unidad Ejecutora en el año 2002 [Archivo de datos]. San José: PANI.

Patronato Nacional de la Infancia, Oficina de Planificación, SII-PANI. (2003, I trimestre). Cantidad de Atenciones realizadas y Omisión de Derechos según: Región y Oficina Local (incluye la Aldea Arthur Gough) [Archivo de datos]. San José: PANI.

Pérez, María Esther. (1999). Manifestaciones de la violencia intrafamiliar en niñas y niños del área rural en el cantón de San Ramón. Tesis para optar por el grado de Magister Scientae en Salud Pública, Escuela de Salud Pública, Universidad de Costa Rica, San José, Costa Rica.

Piaget, Jean. (1932/1974). El criterio moral en el niño. Barcelona: Fontanella.

Piaget, Jean. (1995). Seis estudios de psicología. Colombia: Editorial Labor.

Punch, Samantha. (2002a). Research with Children. The Same or different from research with adults? Childhood, 9 (3), 321-341.

Punch, Samantha. (2002b). Interviewing Strategies with Young People: the 'Secret Box', Stimulus Material and Task-based Activities. Children and Society, 16, 45-56.

República de Costa Rica. (1998). Código de la Niñez y la Adolescencia. Recuperado el 14 de abril de 2004, de http://www.protegiendoles.org/documentacion/articulo23.pdf.

Reyes, Benjamín. (2006) La experiencia de culpa y su relación con la atribución causal del dolor o perjuicio de los demás: un estudio con estafadores psicopáticos y no psicopáticos. Tesis para optar por el grado de Licenciatura en Psicología, Escuela de Psicología, Universidad de Costa Rica, San José, Costa Rica. 
Saavedra, Paulina, Cordero, Maylin, y Calderón, Shirley. (1999). No sólo queremos hablar queremos que nos oigan: Derecho a la participación de niños, niñas y adolescentes. San José: Instituto de Investigaciones Psicológicas, Universidad de Costa Rica.

Simpson, Jeffry A. (1999). Attachment theory in modern evolutionary perspective. En J. Cassidy y P. Shaver (Comps.), Handbook of attachment. Theory, research and clinical applications (pp. 115-140). EE. UU.: The Guilford Press.

Smetana, Judith. (1981). Preschool Children's Conceptions of Moral and Social Rules. Child Development, 52, 1333-1336.

Sorbring, Emma, Rödholm-Funnemark, Margaretha y Palmérus, Kerstin. (2003). Boys' and Girls' Perceptions of Parental Discipline in Transgression Situations. Infant and Child Development, 12, 53-69.

Strauss, Anselm y Corbin, Juliette. (1990). Basics of Qualitative Research: grounded theory procedures and techniques. Newbury Park, California: SAGE.

Strauss, Murray. (1994). Beating the devil out of them. Corporal punishment in American Families. EE. UU.: Lexington Books.

Thoits, Peggy A. (1989). The Sociology of Emotions. Annu. Rev. Sociol., 15, 317-342.

Torres Henríquez, Gabriel. (1996). Desarrollo Moral: un aporte Teórico-Práctico para un Desafío Educativo. Revista Perspectiva Educacional, (27), 119-132.

UNICEF. (2008). VI Estado de los derechos de la niñez y la adolescencia en Costa Rica. Recuperado el 10 de enero de 2008 de http://www.ucr.ac.cr/documentos/EDNA2008.pdf.

Valles Martínez, Miguel. (2003). Técnicas cualitativas de investigación social: reflexión metodológica y práctica profesional. Madrid: Editorial Síntesis.

Valtin, Renate y Walper, Sabine. (1996). Lo que los niños piensan sobre los castigos. Educación, 54, 61-83.

van ljzendoorn, Marinus y Sagi, Abraham. (1999). Cross-cultural patterns of attachment: universal and contextual dimensions. En J. Cassidy y P. Shaver (Comps.), Handbook of attachment. Theory, research and clinical applications (pp. 713-734). EE. UU.: The Guilford Press.

van ljzendoorn, Marinus y Zwart-Woudstra, Hylda A. (1995). Adolescents' Attachment Representations and Moral Reasoning. The Journal of Genetic Psychology, 156 (3), 359-372.

Villegas de Posada, Cristina. (1996). Desarrollo moral y agentes socializadores. Construcción versus socialización. Revista Latinoamericana de Psicología, 28 (3), 523-532. 
Wellman, Henry. (1995). Desarrollo de la teoría del pensamiento en los niños. Bilbao: Desclée de Brouwer.

Williams, Norman y Williams, Sheila. (1976). Desarrollo moral del niño. México D.F.: Publicaciones Culturales.

Zúñiga, María de los A. y Carballo, José A. (2003). La familia costarricense de cara al nuevo siglo. Heredia: Instituto de Estudios Sociales en población (IDESPO), Universidad Nacional. 